\title{
First-trimester combined screening for trisomy 21 in a predominantly Chinese population
}

\author{
T. Y. LEUNG*, L. W. CHAN*, T. N. LEUNG*, T. Y. FUNG*, D. S. SAHOTA*, K. SPENCER† and \\ T. K. LAU* \\ "Department of Obstetrics and Gynecology, The Chinese University of Hong Kong, Hong Kong, China SAR and †Prenatal Screening Unit, \\ Clinical Biochemistry Department, Harold Wood Hospital, Romford, Essex, UK
}

KEYWORDS: Chinese; free $\beta$-hCG; nuchal translucency; PAPP-A; screening; trisomy 21

\begin{abstract}
Objective To examine the effectiveness of first-trimester fetal trisomy 21 screening using a combination of maternal age, nuchal translucency thickness (NT) and maternal serum free beta-human chorionic gonadotropin ( $\beta-h C G)$ and pregnancy-associated plasma protein-A (PAPP-A) levels in a predominantly Chinese population in Hong Kong.
\end{abstract}

Methods This was a prospective study over a 1.5year period of 2990 women who underwent combined screening for trisomy 21 between $11+0$ and $13+$ 6 weeks of gestation in a university fetal medicine unit. NT was measured according to the criteria set by The Fetal Medicine Foundation (FMF), maternal serum free $\beta-h C G$ and PAPP-A levels were measured, and the risk of trisomy 21 was calculated using The FMF's algorithm. Fetal karyotyping was advised when the risk was $1: 300$ or above. All subjects were followed up for pregnancy and fetal outcome.

Results Of the 2990 women who underwent the screening program, 99\% were Chinese. There were 57 twin pregnancies, giving a total of 3047 fetuses. Thirty-one percent of the women were 35 years old or above. One hundred and eighty-five (6.1\%) fetuses were screenpositive; this included 14 cases of trisomy 21 and 17 cases of other chromosomal abnormalities. The positive predictive value was $16.7 \%$. Among the 2862 screennegative fetuses, only $18(0.6 \%)$ cases had an unknown fetal outcome. There were no cases in which trisomy 21 was missed and the infant was liveborn.

Conclusion First-trimester combined screening for fetal trisomy 21 is highly effective among Chinese subjects.
Copyright (C) 2006 ISUOG. Published by John Wiley \& Sons, Ltd.

\section{INTRODUCTION}

Over the last two decades, there have been many major developments in prenatal screening for trisomy 21. Although for years, maternal age was used as the sole screening test for trisomy 21 , it is now clear that such an approach is ineffective when compared with many other tests that are available nowadays. Of all the one-stage screening tests, first-trimester combined nuchal translucency thickness (NT) and biochemical screening using free beta-human chorionic gonadotropin ( $\beta$-hCG) and pregnancy-associated plasma protein-A (PAPP-A) at 11-14 weeks of gestation appears to be the most effective. It has the highest sensitivity at the same false-positive rate $^{1}$, and the sensitivity is comparable to that of two-stage integrated screening ${ }^{2,3}$. Previous studies have shown that the majority of pregnant women prefer first-trimester to later screening ${ }^{4,5}$. This preference is undoubtedly related to the perceived physical, psychological and social benefits of early screening, diagnosis and intervention.

However, most studies on first-trimester screening have been performed in populations of predominantly Caucasian women, despite the fact that it is well known that there are major biological differences between ethnic groups which could significantly affect the performance of a screening test. For example, previous studies have shown that, when compared with Caucasians, Chinese women have significantly higher median values for the first-trimester biochemical markers ${ }^{6,7}$. The aim of this study was to report our initial experience of 2990 cases of combined first-trimester screening tests in a predominantly Chinese population.

Correspondence to: Dr T. Y. Leung, Department of Obstetrics and Gynaecology, Prince of Wales Hospital, Shatin, Hong Kong, China SAR (e-mail: tyleung@cuhk.edu.hk)

Accepted: 4 May 2006 


\section{METHODS}

This was a prospective interventional study of women attending the first-trimester combined screening program for Down syndrome in a university hospital between June 2003 and November 2004. The Fetal Medicine Foundation (FMF)'s, screening program protocols, including case selection, measurement of NT and biochemical analysis, were followed strictly (www.fetalmedicine.com). Briefly, the test was performed only in singleton or twin pregnancies between $11+0$ and $13+6$ weeks' gestation (77-97 gestational days). Fetal crown-rump length (CRL) and NT were measured sonographically (HDI 5000 or HDI 3000 ultrasound machines, Philips Medical System, Amsterdam, The Netherlands) by FMF-accredited doctors.

Maternal blood samples were taken at the same visit, and the levels of free $\beta$-hCG and PAPP-A were measured immediately at the clinic using a Kryptor analyser (Brahms Diagnostica GmbH, Berlin, Germany). The laboratory is accredited by The FMF, and has also joined the quality-control program of the United Kingdom National External Quality Assessment Service (UKNEQAS) with consistently good results. Maternal ethnicity, age, weight, height and smoking status were also recorded at the time of screening.

The risk of trisomy 21 was calculated using The FMF's algorithm and software. In cases of twin pregnancy, a risk was calculated for each fetus based on the individual NT and the maternal serum biochemistry corrected for twin pregnancies. For monoamniotic twin pairs, the highest calculated risk among the co-twins was used.

A risk of 1:300 or greater was regarded as screenpositive and an invasive diagnostic procedure such as chorioinic villus sampling was offered. All fetal outcomes were followed up for any false-negative cases and complications.

Previous studies have shown that there are ethnic differences in maternal serum levels of both PAPP-A and free $\beta-\mathrm{hCG}^{6,7}$, which was not taken into account in the version of The FMF's software used during the study period. Therefore, the risk values were recalculated retrospectively after adjustment with Chinese-specific multiples of the median (MoM) for both hormones, using our previously published formula ${ }^{6}$. The results were then compared with those produced by The FMF's software. The Statistical Package for Social Sciences for Windows, version 13.0 (SPSS Inc, Chicago, IL, USA), was used for statistical analysis of all data. A $P$-value of $<0.05$ was considered statistically significant.

\section{RESULTS}

During the study period, 2990 women underwent the screening program. The ethnic origin of the participants was predominantly Chinese $(n=2953(99 \%))$; there were 14 Caucasian women and 23 were from other Asian countries. Fifty-seven women had a twin pregnancy and hence there were 3047 fetuses in total. The median maternal age was 33 years and $31 \%$ of the women were $\geq 35$ years. The median gestational age at firsttrimester screening was $12+4$ (range, $11+0$ to $13+6$ ) weeks.

The outcome of all cases is summarized in Figure 1. There were $185(6.1 \%)$ screen-positive cases, including seven of the 114 twin fetuses (in 5/57 twin pregnancies), and 180 screen-positive cases were Chinese. All except three $(98.4 \%)$ underwent an invasive diagnostic procedure. Fourteen cases of trisomy 21 were identified, including one from a dizygotic twin pregnancy. The calculated risk for these 14 cases using The FMF's software ranged from $1: 3$ to $1: 181$. In addition, there were 17 other chromosomal abnormalities detected (trisomy $18, n=8$; trisomy $13, n=3$; Turner syndrome, $n=3$; $47, \mathrm{XXX}, n=1$; two cases of other major abnormalities: 46XY,12p+ and 46XY,der(6)t(6;7)(q26;q31.2)). All of these were Chinese pregnancies. The overall positive predictive value for chromosomal abnormalities was therefore $16.7 \%$. Three $(9.7 \%)$ of these abnormal cases (Turner syndrome, trisomy 18 and 47,XXX) underwent spontaneous miscarriage: the case of Turner syndrome had an NT of $3.6 \mathrm{~cm}$ and reversed flow in the ductus venosus; the case of trisomy 18 had a huge NT of $8 \mathrm{~cm}$ and a very low PAPP-A level of $0.09 \mathrm{MoM}$; the case of 47,XXX also had a low PAPP-A level of 0.2 MoM.

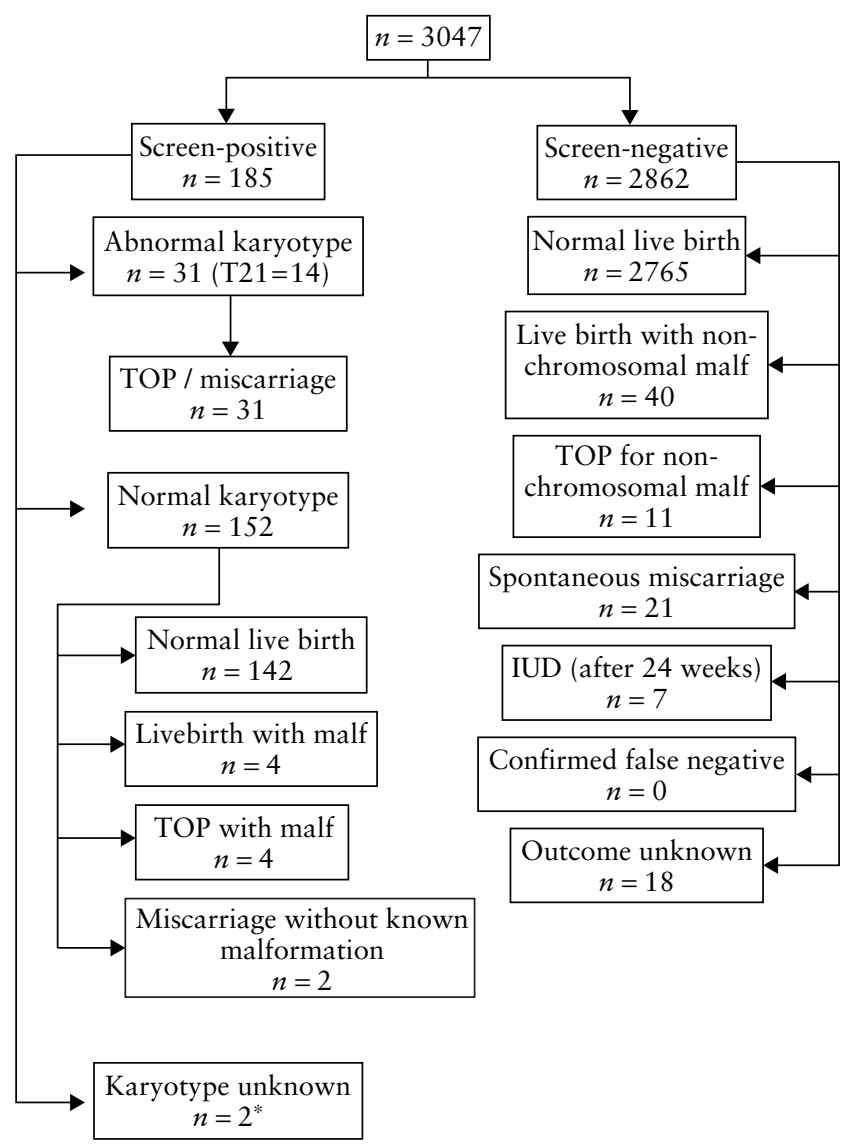

Figure 1 Flow chart showing the outcome of 3047 fetuses that underwent first-trimester combined screening for trisomy 21. "One case of spontaneous miscarriage and one case of IUD. IUD, intrauterine death; malf, malformation; T21, trisomy 21; TOP, termination of pregnancy. 
The rest of the cases with chromosomal abnormalities underwent termination of pregnancy (TOP).

Of the three screen-positive cases which did not undergo an invasive procedure, two miscarried before the scheduled procedure. The karyotype was confirmed as normal by placental biopsy in one of these two, but remained unknown in the other. In the third case an invasive test was declined but there was pre-eclampsia complicated with intrauterine death (IUD) at 28 weeks. No structural abnormality was found at autopsy and the karyotype remained unknown.

Among the 152 fetuses that were screen-positive and had a normal karyotype, there were eight cases of structural abnormalities. Four of these were liveborn, while the other four were terminated. There were two cases of spontaneous miscarriage $(1.3 \%$, including the one that had a placental biopsy, discussed above). The remaining 142 cases had a normal outcome.

Concerning the 2862 screen-negative cases, fetal outcome was available in all except 18 cases $(0.6 \%)$. There were 51 cases of structural malformation that were unrelated to chromosomal abnormalities; 40 were liveborn and the other 11 pregnancies underwent TOP. There were 21 cases of spontaneous miscarriage and seven cases of IUD. No known features of chromosomal abnormalities were identified among these cases. No case of trisomy 21 was liveborn. Therefore, the sensitivity for detecting trisomy 21 of this screening was $100 \%$ (95\% CI, $78-100 \%$ ).

The medians of the gestation-specific MoMs of free $\beta$-hCG and PAPP-A levels among Chinese cases calculated using The FMF's algorithm were significantly higher than were those calculated using the Chinese-specific formula ${ }^{6}$ (free $\beta$-hCG: 1.14 vs. $1.02, P<0.001$; PAPP-A: 1.22 vs. 1.03, $P<0.001$; Wilcoxon's test). The risk value of fetal trisomy 21 was therefore recalculated using our previously reported Chinese-specific MoMs for the hormones ${ }^{6}$. The overall screen-positive rate then became $6.0 \%$ (181 cases at a cut-off risk of $1: 300$ or above), which was very similar to the pre-adjustment rate; 12 cases which were regarded as screen-negative became positive, while 11 cases changed from positive to negative. When compared with the risk based on The FMF medians, this new risk was increased by more than $5 \%$ in 457 cases, reduced by more than $5 \%$ in 308 cases, and the change was $5 \%$ or less in the remaining 2282 cases. All 14 cases of trisomy 21 remained in the screen-positive group with the readjusted risk ranging from $1: 2$ to $1: 210$.

\section{DISCUSSION}

This study has confirmed that the first-trimester combined screening model developed by The FMF is an effective test in a Chinese population, with $100 \%$ (95\% CI, 78-100\%) sensitivity in detecting fetal Down syndrome at a falsepositive rate of $6.1 \%$. The results are comparable to reported figures from the Caucasian population, with sensitivities of $84 \%$ in Germany ${ }^{8}, 85 \%$ in the USA ${ }^{1}, 90 \%$ in Australia ${ }^{9}$ and $93 \%$ in the $\mathrm{UK}^{10}$.
One of the strengths of this study was the follow-up rate, which was very high compared with other similar studies $^{1,8-10}$. It was possible that some trisomy 21 pregnancies might have ended in spontaneous miscarriage or intrauterine fetal demise without karyotypic confirmation. However, the expected number of fetuses with trisomy 21, calculated using The FMF's algorithm based on the patient characteristics, including maternal and gestational ages, was 13 , a value very similar to the actual number of cases detected $(n=14)$. We therefore believe that the high sensitivity of the test in the cohort was not due to under-diagnosis of affected cases. While we cannot claim a sensitivity of $100 \%$ because of the limited sample size, we are quite confident that the sensitivity of this test among the Chinese population is comparable to that in previous studies, about $90 \%{ }^{1,8-10}$.

Most Down syndrome screening programs are based on adjustment of the a priori risk. It has been speculated that the Chinese or Asian population has a lower incidence of trisomy $21^{11-13}$, and therefore that the use of a priori risk based on Caucasian data might be inappropriate. However, we have shown previously that the incidence of trisomy 21 in our Chinese population, where a clinical diagnosis could be made consistently at birth, was not different from that of a Caucasian population ${ }^{14}$. Previous reports of a significantly lower incidence of trisomy 21 among the Chinese are most likely a result of poor case ascertainment. Our results provide further support that the incidence of trisomy 21 in the Chinese population is not lower than that in the Caucasian population and that the application of a priori risk is appropriate.

Previous studies have suggested that there is no significant difference in fetal NT between Chinese and Caucasian populations ${ }^{7}$. On the other hand, levels of free $\beta$-hCG and PAPP-A among Chinese women were 1.15 and 1.23 times higher than those of Caucasian women, which if taken into account might increase the screen-positive rate from $5.3 \%$ to $5.9 \%{ }^{6,7}$. Our study has confirmed the presence of such a difference, and showed that the algorithm for adjustment was correct because the medians of the post-adjusted MoMs of these two hormones were very close to 1 . However, these adjustments had no effect on the screen-positive rate, which remained at $6 \%$. The effects of the hormones on the adjusted risk values and hence the screen-positive rate was much diminished in this study, probably because of the pre-existing high screenpositive rate, which might have been related to maternal age. Nonetheless, it appears that adjustment may not have a major impact on performance of the screening test, although the calculated individualized risk might be more precise with adjustment.

Data concerning combined screening in twin pregnancies are limited. A higher screen-positive rate and a lower detection rate compared with that in singleton pregnancies was reported in a Caucasian population ${ }^{15}$. The higher screen-positive rate is secondary to a higher prevalence of increased NT in twin fetuses, especially among monochorionic twins ${ }^{16}$. The two first-trimester biochemical markers only add a further $5 \%$ to the detection rate 
compared with using NT alone. While there seems to be no significant difference in biochemical levels between monochorionic and dichorionic twins ${ }^{17}$, no data concerning the biochemical levels in Chinese twin pregnancies are available yet. In our study, the screen-positive rates between singleton and twin pregnancies were very close $(6.1 \%)$, and the single case of trisomy 21 in twins was detected. However, a large study population is required to investigate the effectiveness of the screening program in Chinese twin pregnancies.

We believe that the extremely high sensitivity of this study was because of the high standard of quality control, including our compliance to regular external quality assessment by The FMF and UKNEQAS. Additionally, all data in this study came from a single center, which makes quality control much easier. This high standard of quality control must be maintained when the screening program is to be implemented in other Chinese populations.

Over $98 \%$ of women who were screen-positive decided to have a diagnostic test. This is very different from the results of studies of predominantly Caucasian populations, in which only $62 \%$ had a diagnostic test ${ }^{9}$. We believe that this was probably related to the way in which the result was released. Although we do provide the actual adjusted risk to all women, we also explained that we used $1: 300$ as a cut-off and therefore they were classified as high risk or low risk accordingly. Being labeled as 'high-risk' might have affected the decision of our patients.

The spontaneous miscarriage rate in our study was $9.7 \%$ among screen-positive chromosomally abnormal cases after the invasive procedure, $1.3 \%$ among screenpositive chromosomally normal cases after the invasive procedure, and $0.7 \%$ for screen-negative cases that did not undergo an invasive procedure. The increase in the risk of miscarriage after an invasive procedure was about $0.6 \%$, which was very similar to that reported in another study from the same center ${ }^{18}$. These figures may also imply that the chance of spontaneous miscarriage among chromosomally abnormal cases is about $9 \%$. Although the number of cases in our study was limited, this finding is consistent with that reported by Snijders et al. ${ }^{19}$.

In conclusion, first-trimester combined screening for fetal trisomy 21 in the Chinese population is as effective as it is in Caucasian populations, with high sensitivity and low false-positive and high positive predictive rates. Maternal levels of free $\beta$-hCG and PAPP-A are higher in the Chinese population and adjustment is required in the estimation of risk.

\section{REFERENCES}

1. Malone FD, Canick JA, Ball RH, Nyberg DA, Comstock CH, Bukowski R, Berkowitz RL, Gross SJ, Dugoff L, Craigo SD, Timor-Tritsch IE, Carr SR, Wolfe HM, Dukes K, Bianchi DW, Rudnicka AR, Hackshaw AK, Lambert-Messerlian G, Wald NJ, D'Alton ME. First- and Second-Trimester Evaluation of Risk (FASTER) Research Consortium. First-trimester or secondtrimester screening, or both, for Down's syndrome. N Engl J Med 2005; 353: 2001-2011.
2. Spencer K, Souter V, Tul N, Snijders R, Nicolaides KH. A screening program for trisomy 21 at $10-14$ weeks using fetal nuchal translucency, maternal serum free $\beta$ hCG and PAPP-A. Ultrasound Obstet Gynecol 1999; 13: 231-237.

3. Spencer K, Spencer CE, Power M, Dawson C, Nicolaides KH. Screening for chromosomal abnormalities in the first trimester using ultrasound and maternal serum biochemistry in a onestop clinic: a review of three years prospective experience. BJOG 2003; 110: 281-286.

4. de Graaf IM, Tijmstra T, Bleker OP, van Lith JM. Women's preference in Down syndrome screening. Prenat Diagn 2002; 22: 624-629.

5. Spencer K, Aitken D. Factors affecting women's preference for type of prenatal screening test for chromosomal anomalies. Ultrasound Obstet Gynecol 2004; 24: 735-739.

6. Leung TY, Spencer K, Leung TN, Fung TY, Lau TK. Higher median levels of free beta-hCG and PAPP-A in the first trimester of pregnancy in a Chinese ethnic group. Implication for first trimester combined screening for Down's syndrome in the Chinese population. Fetal Diagn Ther 2006; 21: 140-143.

7. Spencer K, Heath V, El-Sheikhah A, Ong CY, Nicolaides KH. Ethnicity and the need for correction of biochemical and ultrasound markers of chromosomal anomalies in the first trimester: a study of Oriental, Asian and Afro-Caribbean populations. Prenat Diagn 2005; 25: 365-369.

8. von Kaisenberg CS, Gasiorek-Wiens A, Bielicki M, Bahlmann F, Meyberg H, Kossakiewicz A, Pruggmayer M, Kamin G, Fritzer E, Harris C, Arnold N. German Speaking Down Syndrome Screening Group. Screening for trisomy 21 by maternal age, fetal nuchal translucency and maternal serum biochemistry at 11-14 weeks: a German multicenter study. J Matern Fetal Neonatal Med 2002; 12: 89-94.

9. Hadlow NC, Hewitt BG, Dickinson JE, Jacoby P, Bower C. Community-based screening for Down's Syndrome in the first trimester using ultrasound and maternal serum biochemistry. BJOG 2005; 112: 1561-1564.

10. Avgidou K, Papageorghiou A, Bindra R, Spencer K, Nicolaides KH. Prospective first-trimester screening for trisomy 21 in 30,564 pregnancies. Am J Obstet Gynecol 2005; 192: 1761-1767.

11. Rogers MS. Racial variations in the incidence of trisomy 21. $\mathrm{Br}$ J Obstet Gynaecol 1986; 93: 597-599.

12. Hoe TS, Boo NY, Clyde MM. Incidence of trisomy 21 in a large Malaysian maternity hospital over an 18 month period. Singapore Med J 1989; 30: 246-248.

13. Higurashi $M$, Oda $M$, Iijima $K$, Iijima $S$, Takeshita $T$, Watanabe N, Yoneyama K. Livebirth prevalence and follow-up of malformation syndromes in 27,472 newborns. Brain Dev 1990; 12: 770-773.

14. Lau TK, Fung HY, Rogers MS, Cheung KL. Racial variation in incidence of trisomy 21: survey of 57,742 Chinese deliveries. Am J Med Genet 1998; 75: 386-388.

15. Spencer K, Nicolaides KH. Screening for trisomy 21 in twins using first trimester ultrasound and maternal serum biochemistry in a one-stop clinic: a review of three years experience. BJOG 2003; 110: 276-280.

16. Spencer K. Screening for trisomy 21 in twin pregnancies in the first trimester using free beta-hCG and PAPP-A, combined with fetal nuchal translucency thickness. Prenat Diagn 2000; 20: 91-95.

17. Sebire NJ, Snijders RJM, Hughes K, Sepulveda W, Nicolaides KH. Screening for trisomy 21 in twin pregnancies by maternal age and fetal nuchal translucency thickness at 10-14 weeks of gestation. Br J Obstet Gynaecol 1996; 103: 999-1003.

18. Lau TK, Leung TY, Fung TY, Chan LW, Sahota DS, Leung TN. Outcome of 1355 consecutive transabdominal chorionic villus samplings in 1351 patients. Chin Med J 2005; 20: $1675-1681$.

19. Snijders RJ, Sebire NJ, Nicolaides KH. Maternal age and gestational age-specific risk for chromosomal defects. Fetal Diagn Ther 1995; 10: 356-367. 